\title{
Mathematical Model of a Cracked Pipeline Subjected to Sinusoidal Force Excitation
}

\author{
Ibrahim M. El Fahham*, Abdalla H. Al Kaood and Hassan A. El Gamal \\ Mechanical Engineering Department, Faculty of Engineering, Alexandria University, Egypt \\ *Corresponding author: ibrahim.elfahham@alexu.edu.eg
}

\begin{abstract}
The present work introduces a mathematical model of a cracked pipeline conveying liquid. This model uses governing equation of Euler - Bernoulli beam theory for a pipe conveying liquid. The crack introduced in the model is represented by two identical torsional springs and a sinusoidal excitation force was applied to one of the pipe ends for the purpose of crack detection. The model is solved numerically using MATLAB code bvp4c to solve linear, ordinary fourth order differential equations (boundary value problem) for the detection of the crack position. To group the variables in the dimensionless form, Buckingham Pi-theorem was used. The effects of the dimensionless parameters on crack position were examined. The results show that the value of dimensionless parameters of stiffness at crack and support has considerable effect on crack position. It also shows that the force amplitude and fluid flow properties has no effect on crack position which demonstrates the capacity of using the present technique with small force magnitude to avoid stress-strain problems on the pipeline and any excitation frequency can be used.
\end{abstract}

\section{Keywords: Mathematical models; Lateral vibration; Pipeline; Crack position; Dimensional analysis}

\section{Introduction}

In order to eliminate loss of water from pipelines many techniques have been proposed. Water companies apply many techniques at the water distribution systems and this helps to know where the cracks are in the network that causes leakage. However, these various methods for detection cannot give the exact information about the position of the crack that causes the leakage in the pipeline. Many researches have been carried out on the detection of crack position.

Housner [1] studied the free and forced vibrations which are produced from the crosswinds and that perhaps cause problems in the pipelines supported above ground. The proposed solution based on simple beam theory, demonstrated that the flow of fluid in the pipeline has negligible effect on the vibrations. For free and steady- state forced vibrations, it was found that when the value of damping is too small, large amplitudes may be developed. Long [2] used analytical and experimental methods for the studding of the free transverse vibrations for the fundamental mode of a single-span pipeline containing a flowing fluid. The approximate solution used an infinite power series to solve the governing differential equation of motion. The results show that, a slight decrease in frequency and no decaying vibration with an increase in flow rate of the flowing fluid for simply supported, fixed, and fixed-simple ends. Stein and Tobriner [3] presented numerical solution to the governing equation of an elastically supported pipe of infinite length carrying an ideal fluid under pressure. Furthermore, the effect of resulting internal pressure forces is demonstrated. The effects of flow velocity, foundation modulus and internal pressure on the dynamic stability, also frequency response, and wave-propagation characteristics of an undamped system are discussed. The discussions show that, the stability of the system is insured if the flow velocity does not exceed the critical value. Weaver [4] studied the dynamic stability of a pipeline with a finite-length carrying a fluid. The equation of Flügge - Kempner is used with classical potential theory in which circumferential and usual beam modes may be considered. The results show that, cylinders become statically unstable at first but flutter is predicted at higher velocity. The critical flow velocities for short thin shells are associated with circumferential waves. Mukherjee and Narasimhan [5] presented a new technique for detection the location and size of the cracks if single or numerous that happens in a pipeline networks. The technique is a combination of the generalized likelihood ratio GLR test, which utilizes volume rate and pressure measurements and a steady- state model of the network. Lee and Chung [6] proposed a new non-linear model of a straight pipeline carrying fluid and the pipeline is clamped from both ends. The governing equations used the Euler -Bernoulli beam theory and non -linear 
Lagrange strain theory, from the extended Hamilton principle the coupled non -linear equations of motion for the axial and lateral vibrations. The governing equations are solved by using Galerkin method. Reddy and Wang [7] introduced governing equations of fluid-conveying beams using two well-known relations; Timoshenko beam theories and Euler Bernoulli. The formulation focus on geometric nonlinearity and influence of fluid velocity to the kinetic energy and body forces with accordance with the Von Karman theory. The model with non-linear equations of motion were presented in Finite element models. Kupier and Metrikine [8] derived theoretically the stability of a clamped-pinned pipe conveying fluid at low fluid flow velocity. The model is an adaptation of a tensioned Euler -Bernoulli beam model and plug flow model. A D-decomposition method was used to study the stability of the pipe. Langre and Paidoussis [9] studied the stability of a thin flexible cylinder and consider it as a beam, when subjected to axial flow and clamped at the upstream end only. The stability is studied by using a finite deference technique to the governing equation of motion in the frequency domain. The linear stability analysis of lateral motion is used to study the flutter effect as a function of the main variables which includes the flow velocity and the cylinder length. Jing and Zhi-Hong [10] studied pipeline leakage factors, using Grey relational analysis in order to analyze the factors and evaluate their degree of influence on pipeline leakage. They put forward a prediction model for the leakage by means of the so called multiple linear regression analysis.

Oikonomidis et al [11] developed a model of prediction for the propagation of crack in pipelines conveying natural gas using a strain rate dependent damage model. The model was adjusted for specific strain rates. Their model was validated by experimental analysis on notched pipe. Zhang et al [12] initiated a prediction code for the leakage from pipeline crack. The model was applied on high and low temperature of single and two phase fluid flow. The model was based on the Henry-Fauske critical flow model and was justified by experimental results of a straight pipe crack. Wang et al [13] studied the emission signal of pipeline crack acoustics. Using a Nielsen-Hsu pencil break method he simulated the fault detection of pipe crack. The precision of position accuracy had an error less than $5 \%$. Kirubakaran and Krishna [14] on the other hand used image analysis for pattern recognition of pipeline crack. The analysis was used to develop a mathematical morphological operator for the detection of cracks edge. Mohamed et al [15] investigated the damage to the pipe caused by crack and erosion corrosion. They found in their analytical analysis, that the solution to the equations for the two cases showed satisfactory agreement with the experimental analysis where the variation of vibrating sensitivity of the measuring sensor detects the presence of defects. They also showed that the vibration of the simply supported pipe in the crack and erosion corrosion cases decreases and increases, respectively. Razvarz et al [16] proposed a new technique for the detection of fluid leaks in pipelines through the evaluation of fluid flow and pressure at the pipe ends. He used a technique based on data combination from steady-state approximation and SEKF second order MATLAB code filter. Finally, Lu et al [17] presented an interesting survey on the novel detection methods used for oil and gas pipelines leakage. His review includes the advantages and limitations of each technique with their appropriate technological tools.

The objective of this paper is to determine the location of crack in a pipeline conveying liquid using mechanical vibration. In order to accomplish this mathematical model was developed to simulate a cracked pipe and the crack is modeled by two torsional springs. A MATLAB program was constructed in order to solve the governing equation of motion simulating the vibrating pipe. Different parameters in their dimensionless forms affecting the crack position are studied. And by the aid of the Buckingham theorem the basic variables of the model are identified.

\section{Governing Equations and Mathematical Modeling}

For the derivation of the governing equation, consider a uniform cross section straight pipe conveying fluid of length $\mathrm{L}$, modulus of elasticity $\mathrm{E}$, cross-section area A and second moment of area I as shown in Figure (1). The fluid in the pipe is at a constant pressure $\mathrm{P}$, density $\rho$, and constant velocity $U$. Let us consider a small element of the pipe with length $\delta \mathrm{x}$. This element is divided into two parts, fluid element and pipe element. An external force $f(x, t)$ is applied on the pipe at a distance $x$ leading to a vertical deformation $\mathrm{w}(\mathrm{x}, \mathrm{t})$. Forces distribution on fluid element and pipe element are as shown in Figure (2).

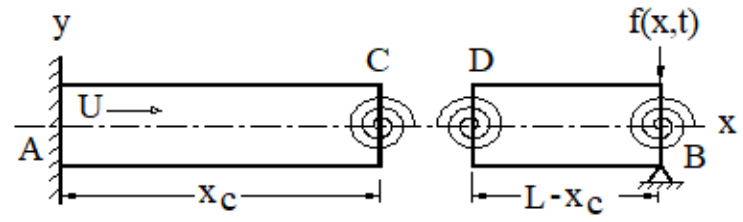

Figure 1- Cracked pipe conveying fluid. 


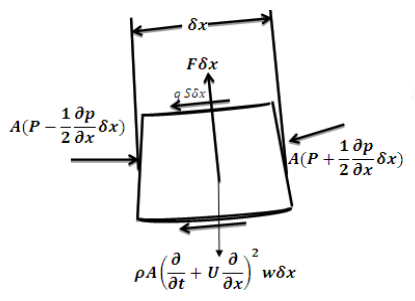

(a) Fluid element

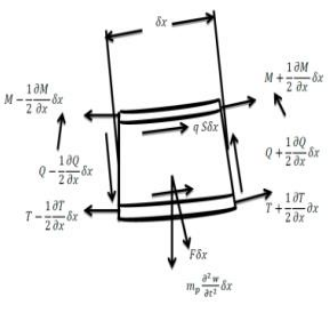

(b) Pipe element Pipe element

The equilibrium of the forces in the $\mathrm{x}$ and $\mathrm{y}$ directions on the fluid element and pipe element gives,

$m_{f} \frac{\partial U}{\partial t}+A \frac{\partial P}{\partial x}+F \frac{\partial w}{\partial x}+q S$

$=0$ (in $x$ direction $)$

$-m_{f}\left(\frac{\partial}{\partial t}+U \frac{\partial}{\partial x}\right)^{2} w+F-A \frac{\partial}{\partial x}\left(P \frac{\partial w}{\partial x}\right)-q S \frac{\partial w}{\partial x}$

$=0 \quad$ (in y direction)

Where $m_{f}$ is the mass of fluid element per unit length of the pipe, $F$ is the fluid force per unit length applied to the fluid element by the tube. $S$ is the inner perimeter of the pipe, $q$ is the shear stress on the internal surface of the pipe and $\mathrm{A}$ is the pipe cross-section area.

The term,

$m_{f}\left(\frac{\partial}{\partial t}+U \frac{\partial}{\partial x}\right)^{2} w=m_{f}\left[\frac{\partial^{2} w}{\partial t^{2}}+U^{2} \frac{\partial^{2} w}{\partial x^{2}}+2 U \frac{\partial^{2} w}{\partial x \partial t}\right]$

represents the inertia force exerted by internal plug

flow on the pipe.

The equations of motion of the pipe element derived in the $\mathrm{x}$ and $\mathrm{y}$ directions are:

$\frac{\partial T}{\partial x}+q S+F \frac{\partial w}{\partial x}=0 \quad$ (in $x$ direction $)$

$\frac{\partial Q}{\partial x}+F-\frac{\partial}{\partial x}\left(T \frac{\partial w}{\partial x}\right)-q S \frac{\partial w}{\partial x}+m_{p} \frac{\partial^{2} w}{\partial t^{2}}+F$

$=f(x, t) \quad($ in $y$ direction $)$

$f(x . t)$ is the external applied force per unit length, $Q$ is the transverse shear force in the pipe, $T$ is the longitudinal tension in the pipe and $m_{p}$ is the mass per unit length of the empty pipe.

The bending moment $M$ in the pipe, the transverse shear force $Q$ and the pipe deformation are related by, $Q=\frac{\partial M}{\partial x}=E I \frac{\partial^{3} w}{\partial x^{3}}$

Where $E$ is the modulus of elasticity of the pipe material and $I$ is the second moment of area of the pipe section.

Combining all the equations above and eliminating $Q$, $q$ and $F$ yields:

$$
\begin{aligned}
E I \frac{\partial^{4} w}{\partial x^{4}}-\frac{\partial}{\partial x}[(T & \left.-P A) \frac{\partial w}{\partial x}\right]+2 m_{f} U \frac{\partial^{2} w}{\partial x \partial t} \\
& +m_{f} U^{2} \frac{\partial^{2} w}{\partial x^{2}}+\left(m_{f}+m_{p}\right) \frac{\partial^{2} w}{\partial t^{2}} \\
& =f(x, t)
\end{aligned}
$$

The fluid pressure causes internal force on the pipe wall. It is possible to change the true longitudinal force due to fixed support in the model (see figure (1)) into a so-called effective tension $T_{\text {eff }}$.

Where,

$$
\begin{gathered}
T_{e f f}=T-P A, \\
m_{t}=m_{p}+m_{f} \text { and }, \\
m_{f}=\rho A, \\
E I \frac{\partial^{4} w}{\partial x^{4}}+\left(m_{f} U^{2}-T_{e f f}\right) \frac{\partial^{2} w}{\partial x^{2}}+2 m_{f} U \frac{\partial^{2} w}{\partial x \partial t} \\
+m_{t} \frac{\partial^{2} w}{\partial t^{2}}=f(x, t)
\end{gathered}
$$

Substituting into equation (7) with the following substitution suitable for harmonic oscillation:

$$
\begin{aligned}
w & =W(x) \cdot e^{i \omega t} \\
f & =F(x) \cdot e^{i \omega t}
\end{aligned}
$$

Where $\omega$ is the frequency of the oscillation,

$E I \frac{d^{4} w}{d x^{4}}+\left(m_{f} U^{2}-T_{e f f}\right) \frac{d^{2} w}{d x^{2}}-\omega^{2} m_{t} w+$

$2 m_{f} U \cdot i \cdot \omega \frac{d w}{d x}=F(x)$

Equation of motion (8) can be made dimensionless by introducing the following variables:

$$
\begin{aligned}
& w^{*} \frac{w}{D_{i}} \text { and } x^{*}=\frac{x}{L} \\
& E I \frac{d^{4} w^{*}}{d x^{* 4}}\left(\frac{D_{i}}{L^{4}}\right)+\left(m_{f} U^{2}-T_{e f f}\right) \frac{d^{2} w^{*}}{d x^{* 2}}\left(\frac{D_{i}}{L^{2}}\right) \\
& -\omega^{2} m_{t} w^{*}\left(D_{i}\right)+2 m_{f} U i \omega \frac{d w^{*}}{d x^{*}}\left(\frac{D_{i}}{L}\right) \\
& =F\left(x^{*}\right) \\
& \frac{d^{4} w^{*}}{d x^{* 4}}+\left(\frac{L^{2} T_{e f f}}{E I}\right)\left(\frac{m_{f} U^{2}}{T_{e f f}}-1\right) \frac{d^{2} w^{*}}{d x^{* 2}} \\
& +\left(\frac{2 m_{f} U L^{3} \omega}{E I}\right) i \frac{d w^{*}}{d x^{*}} \\
& -\left(\frac{\omega^{2} L^{4} M_{t}}{E I}\right) w^{*}=\frac{L^{4} F\left(x^{*}\right)}{E I \cdot D_{i}}
\end{aligned}
$$

Where the primes denote differentiation w. r. t. $x^{*}$ Introducing the following dimensionless parameters:

$$
\beta=\frac{L^{2} T_{\text {eff }}}{E I}, \quad S_{t}=\frac{\omega L}{U}, \quad r=\frac{m_{t}}{m_{f}},
$$




$$
\begin{aligned}
& F^{*}\left(x^{*}\right)=\frac{F(x)}{E I\left(D_{i} / L^{4}\right)}, \quad V=\frac{m_{f} U^{2} L^{2}}{E I} \\
& W^{*^{\prime \prime \prime \prime}}+(V-\beta) W^{*^{\prime \prime}}+2 S_{t} V i W^{*^{\prime}}-r S_{t}^{2} V W^{*}= \\
& F^{*}\left(x^{*}\right)
\end{aligned}
$$

The boundary conditions at the left and the right hand supports of the pipe are,

$$
\begin{gathered}
W^{*}(0)=0, W^{* \prime}(0)=0, W^{*}(1)=0 \\
W^{*^{\prime \prime}}(1)=-K_{2} W^{*^{\prime}}(1)
\end{gathered}
$$

Where,

$$
K_{2}=\frac{K_{r l} L}{E I}
$$

$K_{r l}$ is the spring stiffness at the right support.

The boundary conditions at point $\mathrm{C}$ (crack) are,

$$
W^{*^{\prime \prime}}\left(x_{c}^{*}\right)=-K_{1} W^{*^{\prime}}\left(x_{c}^{*}\right) \text { and } W^{*^{\prime \prime \prime}}\left(x_{c}^{*}\right)=0
$$

Where,

$$
K_{1}=\frac{K_{r c} L}{E I}
$$

$K_{r c}$ is the spring stiffness at the crack and $x_{c}^{*}$ is the dimensionless distance from the left support of the pipe to the crack.

The boundary conditions at point $\mathrm{D}$ (crack) are:

$$
W^{* \prime \prime}\left(x_{c}^{*}\right)=K_{1} W^{* \prime}\left(x_{c}^{*}\right)
$$

and,

$$
W^{*^{\prime \prime \prime}}\left(x_{c}^{*}\right)=0
$$

Where,

$$
K_{1}=\frac{K_{r c} L}{E I}
$$

\section{Numerical Results and Discussions}

To predict a crack location in a pipe conveying liquid a technique is developed based on an external exciter to give the pipe a forced frequency placed nearly at any end of the pipe. This excitation force can be represented as follows:

$$
f(x)=\left[x e^{\left(1-\frac{a}{1-x}\right)}+10^{-40}\right] / f_{\max }
$$

When the exciter is at the left support of the pipe (the blue line in figure 3 )

$$
f(x)=\left[(1-x) e^{\left(1-\frac{a}{x}\right)}+10^{-40}\right] / f_{\max }
$$

When the exciter is at the right support of the pipe (the red dotted line in figure 3 )

Where $f_{m x a}$ is the maximum value attained by the function $f(x)$. The constant $a=50$ is taken to make the exciting force as sharp as possible and the vanishingly small constant $10^{-40}$ in the force equation was put to provide continuity of the exciting signal over the whole span of the pipe as shown in figure (3).

The Algorithm used considers the cracked pipe as two segments each has its boundary conditions. The MATLAB code bvp4c was used to solve the present linear ordinary fourth order differential equations (boundary value problem). The effect of the main dimensionless parameters of the model $\mathrm{V}, \mathrm{S}_{\mathrm{t}}, \mathrm{r}$ , $K_{1}, K_{2}, \beta$, on the crack position $\frac{x_{c}}{L}$ were examined, and found to have in general no sensible effect on crack position, so there is a need to group the basic variables of the governing equations into new groups to examine their mutual effect on the crack position . For this purpose, Dimensional analysis using Buckingham Pi theorem is to be used.

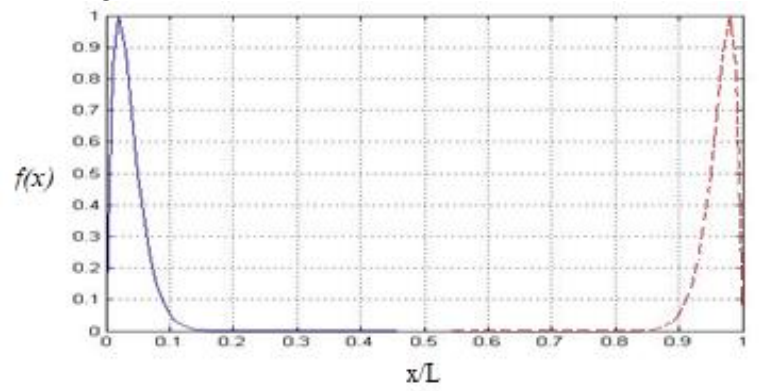

Figure 3- Shape of the excitation force

\subsection{Dimensional Analysis using Buckingham Pi theorem.}

Many steps will have to be done to eliminate the variables in the problem, and the next tables represent the primary dimensions of the system, Table (1) shows the system parameters and primary dimensions. The developed dimensionless new ten parameters ( $\mathrm{Pi}$-groups) are shown in Table (2).

Table 1- System parameters primary dimensions

\begin{tabular}{|l|c|c|c|c|c|c|c||}
\hline Parameter & $w$ & $D_{i}$ & $E$ & $m_{f}$ & $T_{\text {eff }}$ & $F$ & $\omega$ \\
\hline $\begin{array}{l}\text { Primary } \\
\text { Dimensions }\end{array}$ & $L$ & $L$ & $M L^{-1} T^{-}$ & $M L^{-1}$ & $M L T^{-2}$ & $M T^{-2}$ & $T^{-1}$ \\
\hline \hline Parameter & $L$ & $I$ & $K_{r l}$ & $\rho_{s}$ & $K_{r c}$ & $U$ & \\
\hline $\begin{array}{l}\text { Primary } \\
\text { Dimensions }\end{array}$ & $L$ & $L^{4}$ & $M L^{2} T^{-2}$ & $M L^{-3}$ & $M L^{2} T^{-2}$ & $L T^{-2}$ & \\
\hline
\end{tabular}

Table 2- Pi's expressions

\begin{tabular}{|c|c|c|c|c||}
\hline$\pi 1$ & $\pi 2$ & $\pi 3$ & $\pi 4$ & $\pi 5$ \\
\hline$\frac{W}{L}$ & $\frac{D_{i}}{L}$ & $\frac{E}{\rho_{s} U^{2}}$ & $\frac{m_{f}}{\rho_{s} L^{2}}$ & $\frac{T_{e f f}}{\rho_{s} L^{2} U^{2}}$ \\
\hline \hline$\pi 6$ & $\pi 7$ & $\pi 8$ & $\pi 9$ & $\pi 10$ \\
\hline$\frac{F}{\rho_{s} L U^{2}}$ & $\frac{\omega L}{U}$ & $\frac{I}{D d_{i}^{4}}$ & $\frac{K_{r c}}{\rho_{s} L^{3} U^{2}}$ & $\frac{K_{r l}}{\rho_{s} L^{3} U^{2}}$ \\
\hline
\end{tabular}




\subsection{Parameters evaluation}

Figures $(4,5,6$, and 7$)$ show the effect of the initial tension parameter $\left(T_{e f f} / \rho_{S} L^{2} U^{2}\right)$, flow parameter $\left(m_{f} / \rho_{s} L^{2}\right)$, Strouhal number or (frequency parameter) $S_{t}$, and excitation force parameter $\left(F / \rho_{s} L U^{2}\right)$ on crack position $\left(x_{c} / L\right)$ respectively. According to the graphs there is no effect for the parameters on crack position prediction. The predicted crack position was 0.91 when the values of the parameters were as follows: Initial tension parameter $=0.4$, flow parameter $=1\left(10^{4}\right)$, Strouhal number or (frequency) parameter $=65$, excitation force parameter $=0.0001$, elasticity parameter $=2\left(10^{6}\right)$, second moment of area parameter $=0.05$, diameter parameter $=0.02$, crack stiffness parameter $=0.0003$, support stiffness parameter $=0.0004$.

The predicted crack position was 0.55 when the values of the parameters were as follows:

Initial tension parameter $=0.4, \quad$ flow parameter $=1\left(10^{4}\right)$, Strouhal number or (frequency) parameter $=65$, excitation force parameter $=0.0001$, elasticity parameter $=2\left(10^{6}\right)$, second moment of area parameter $=0.05$, diameter parameter $=0.02$, crack stiffness parameter $=2.5$, support stiffness parameter $=0.4$. For $\mathrm{S}_{\mathrm{t}}$ the results mean that in practical applications, the exciter can apply a force to the pipe with any frequency to predict the crack position.

It can easily be inferred from the figures that these four parameters do not have any effect on the predicted location of the crack. In fact, several trials have been carried out for different values of the four parameters in order to examine their mutual effects on the crack location. The procedure was repeated while keeping the rest of the parameters constant. It was found after a process of elimination that the most influential parameters are the crack stiffness parameter $\left(K_{r c} / \rho_{s} L^{3} U^{2}\right)$ and the support stiffness parameter $\left(K_{r l} / \rho_{s} L^{3} U^{2}\right)$. It is quite clear from Figures 4, 5, 6 and 7 that the predicted position of crack changes only when changing those parameters. Two numerical examples are chosen to be plotted on these figures giving a predicted crack position of 0.91 for relatively small values of $\left(K_{r c} / \rho_{s} L^{3} U^{2}\right)=$ 0.0003 and $\left(K_{r l} / \rho_{s} L^{3} U^{2}\right)=0.0004$ and a position of 0.55 for relatively large values of $\left(K_{r c} / \rho_{s} L^{3} U^{2}\right)=$ 2.5 and $\left(K_{r l} / \rho_{s} L^{3} U^{2}\right)=0.4$. The numerical examples shown in the four figures are for values of $\left(D_{i} / L\right)=0.02,\left(I / D_{i}^{4}\right)=0.05$ and $\left(E / \rho_{s} U^{2}\right)=$ $2\left(10^{6}\right)$. On the other hand, it is clear from Figures $(8$, 9) that the effect of $\left(I / D_{i}^{4}\right)$ and $\left(E / \rho_{s} U^{2}\right)$ on the crack position $\left(x_{c} / L\right)$ is quite small. The values of the second moment of area parameter were taken from 0.01 to 0.5 and the predicted position of the crack is seen to vary approximately from 0.98 to 0.9 . The values of the elasticity parameter were taken from $0.1\left(10^{6}\right)$ to $2.5\left(10^{6}\right)$ and the predicted position of the crack varied from approximately 0.91 to 0.98 .

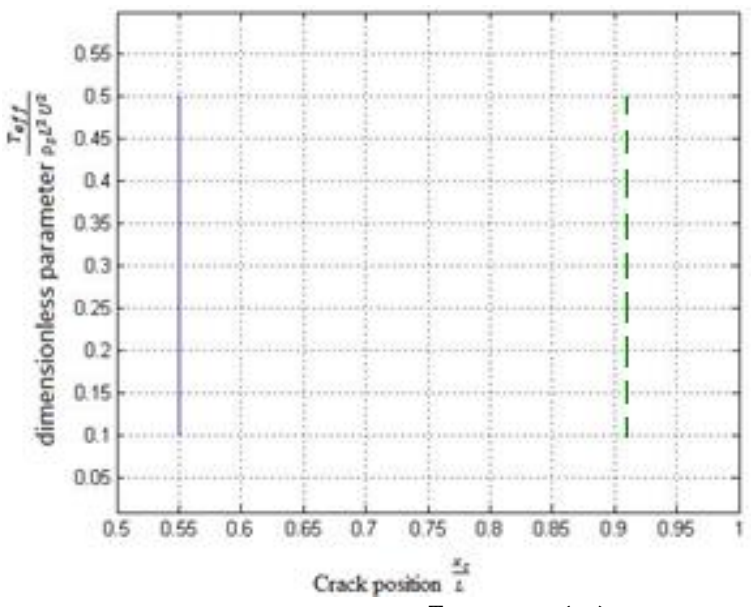

Figure 4- Effect of $\frac{\mathrm{T}_{\mathrm{eff}}}{\rho_{\mathrm{s}} \mathrm{L}^{2} \mathrm{U}^{2}}$ on $\left(\frac{x_{c}}{L}\right)$

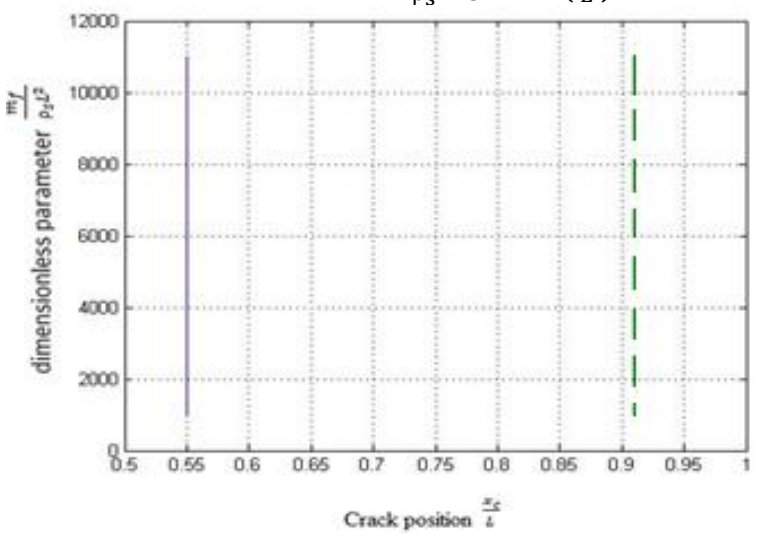

Figure 5- Effect of $\frac{m_{f}}{\rho_{s} L^{2}}$ on $\frac{x_{c}}{L}$

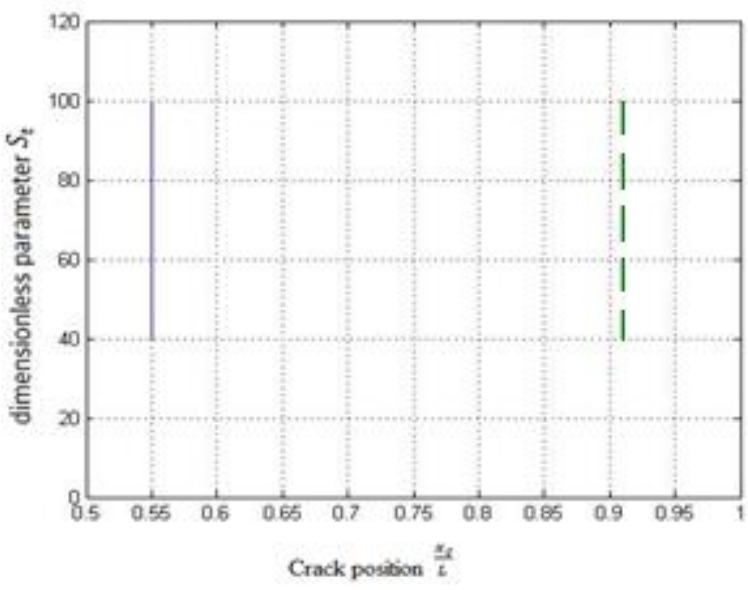

Figure 6- Effect of $S_{t}$ on $\left(\frac{x_{c}}{L}\right)$ 


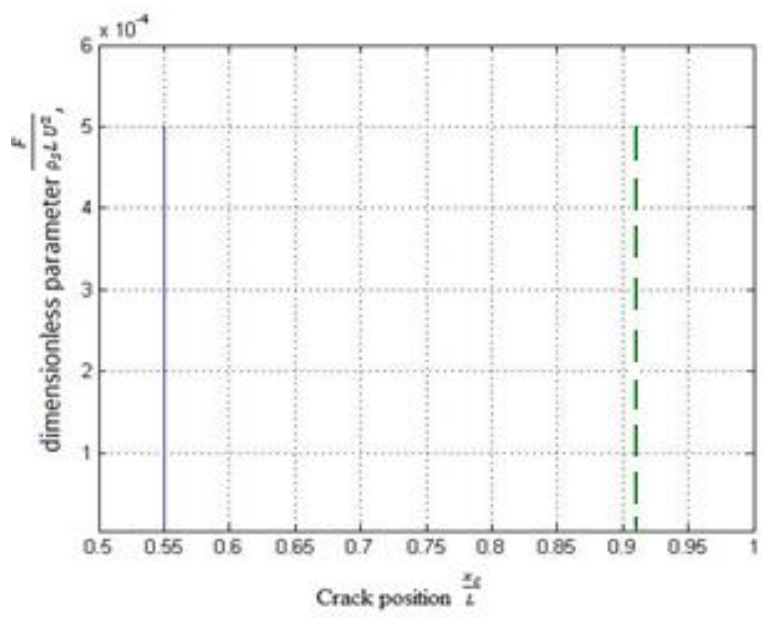

Figure 7- Effect of $\left(\frac{F}{\rho_{s} L U^{2}}\right)$ on $\left(\frac{x_{c}}{L}\right)$

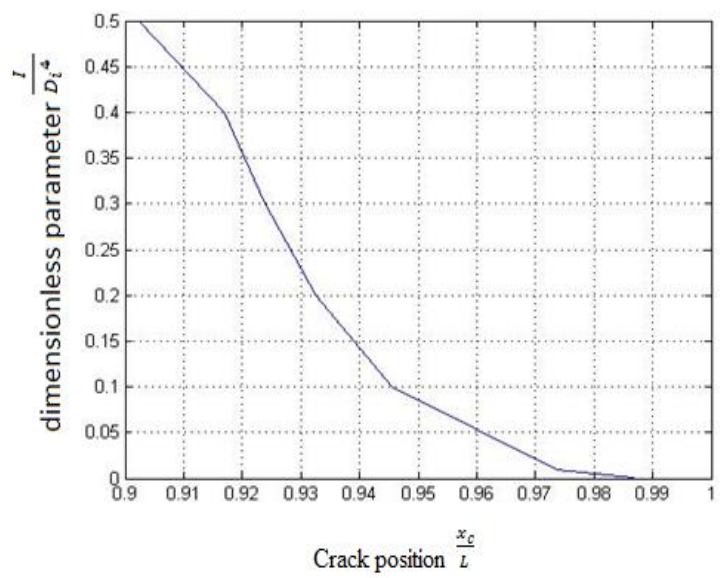

Figure 8- Effect of $\left(\frac{I}{D_{i}^{4}}\right)$ on $\left(\frac{x_{c}}{L}\right)$

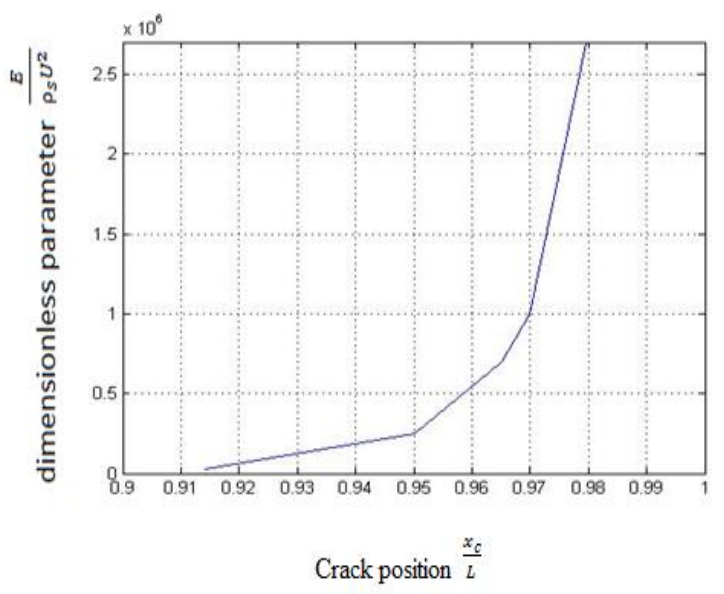

Figure 9- Effect of $\left(\frac{E}{\rho_{S} U^{2}}\right)$ on $\left(\frac{x_{c}}{L}\right)$

Figures $(10,11,12)$ show the effect of diameter parameter $\left(D_{i} / L\right)$, crack stiffness parameter $\left(K_{r c} / \rho_{s} L^{3} U^{2}\right)$, and support stiffness parameter $\left(K_{r l} / \rho_{s} L^{3} U^{2}\right)$ on crack position $\left(x_{c} / L\right)$ respectively.
It can be clearly seen that there is a considerable effect of these parameters on the crack position. For $\left(D_{i} / L\right)$ parameter and according to figure $(10)$ as the values of the parameter increase the technique sensitivity for the crack position prediction increases. The values of the parameter were taken from 0.15 to 0.5 and the predicted position of the crack was 0.95 to 0.6 approximately. And for $\left(K_{r c} / \rho_{s} L^{3} U^{2}\right)$, $\left(K_{r l} / \rho_{s} L^{3} U^{2}\right)$ parameters, when the stiffness of the crack and the support increase the crack position moves close to the middle of the pipe. This means that as the position goes close to the middle of the pipe, the stiffness of the crack and support attain larger values indicating that the crack size is smaller. When the values of the stiffness of crack and support are small, the position moves away from the middle of the pipe meaning that the size of crack is larger. The values of the crack stiffness parameter were taken from 1 to 3.75 and the predicted position of the crack was from 0.97 to 0.6 respectively. The values of the support stiffness parameter were taken from 0.2 to 0.55 and the predicted position of the crack was from 0.95 to 0.5 respectively.

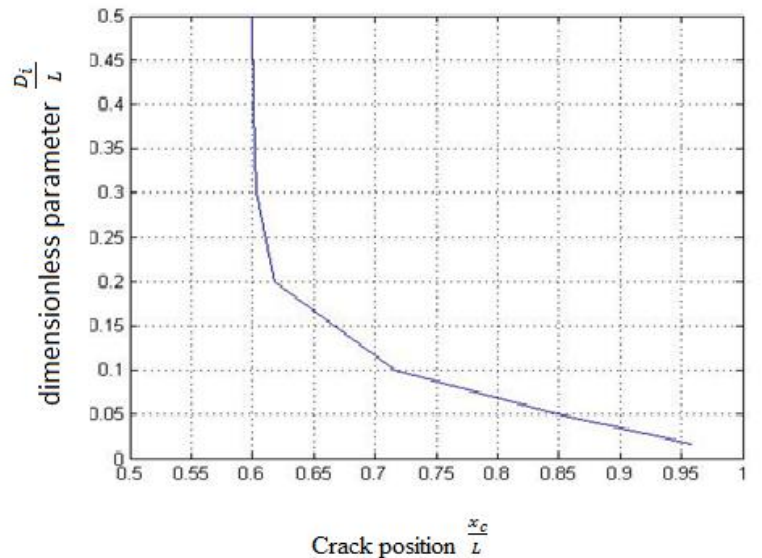

Figure 10- Effect of $\left(\frac{D_{i}}{L}\right)$ on $\left(\frac{x_{c}}{L}\right)$

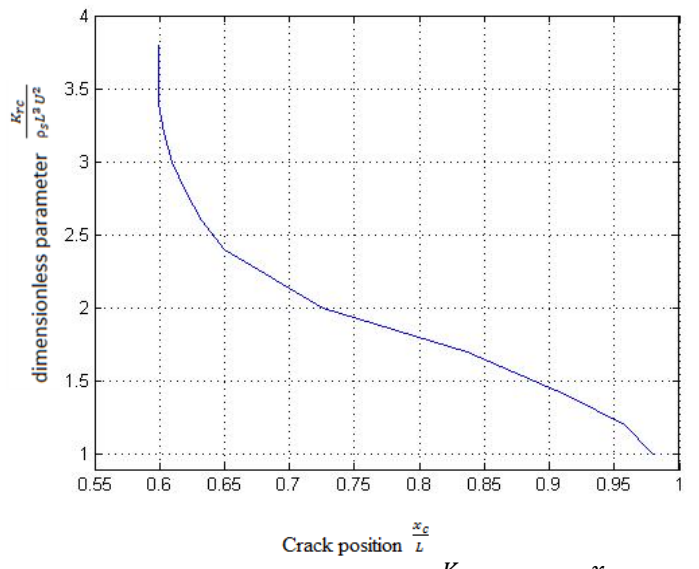

Figure 11- Effect of $\left(\frac{K_{r c}}{\rho_{s} L^{3} U^{2}}\right)$ on $\left(\frac{x_{c}}{L}\right)$ 


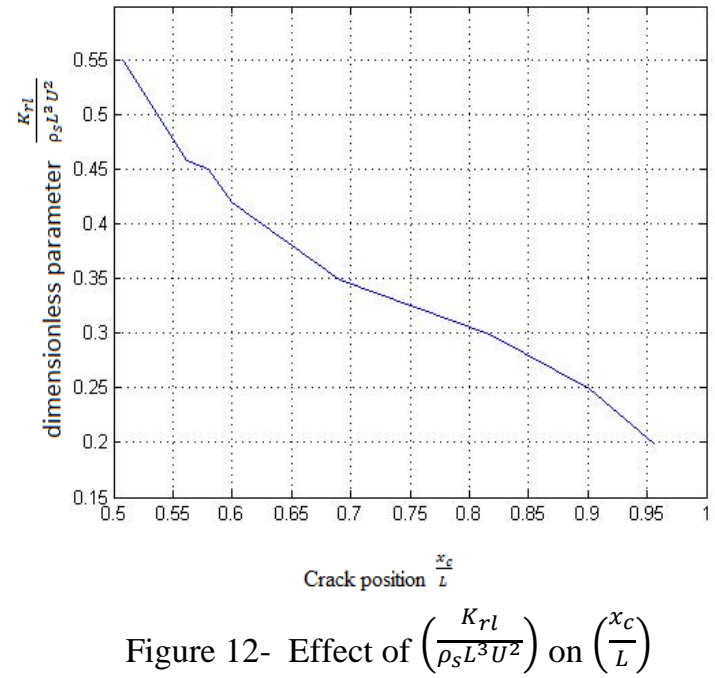

\section{Conclusions}

The present technique introduced a simple method for detecting the crack position in a pipeline with respect to other techniques in this field. The technique can be applied to any pipeline with any supports. It also shows that the force amplitude and fluid flow properties have no effect on crack position which demonstrates the capacity of using the present technique with small force magnitude to avoid stressstrain problems on the pipeline and any excitation frequency can be used. The results show that,

- There are three dimensionless parameters that have considerable effect on the crack position, namely the inner diameter to length parameter, crack stiffness and support stiffness parameters.

- Strouhal number (frequency number) has no sensible effect on crack position prediction, and this result is of advantage to the technique, so there is no restriction on using any frequency without causing any problems to the piping system.

- Crack position prediction depends very much on the type of support.

- Force amplitude has no effect on crack position prediction, and that result is of advantage for the technique, so using small force magnitude is preferable to avoid any problems that may appear to the pipeline.

- Fluid flow properties have limited effect on crack position prediction.

\section{References}

[1] Housner G.W. "Bending vibrations of a pipeline containing flowing fluid" J. applied Mechanics, (1952), Vol.19, pp. 205-208.
[2] Long R.H. "Experimental and theoretical study of transverse vibration of a tube containing flowing fluid " J. Applied Mechanics, (1955), Vol.22, pp. 65-68.

[3] Stein R.A. and Tobriner M.W. "Vibrations of pipes containing flowing fluids" J. applied Mechanics, (1970), Vol. 92, pp. 906-916.

[4] Weaver D.S. "On the dynamic stability of fluid conveying pipes "J. Applied Mechanics, (1973), 40(1), pp. 48-52.

[5] Mukherjee, J. and Narasimhan, S. (1996). Leak detection in networks of pipelines by the generalized likelihood ratio method, Industrial \& Engineering Chemistry Research, ASC 35: pp. 1886-1893.

[6] Lee S.I. and Chung J." New Nonlinear Modelling for Vibration Analysis of a Straight Pipe Conveying Fluid", Sound and Vibration (2002), Vol 254, pp. 313-325.

[7] Reddy J. N. and Wang C. M.," Dynamics of fluid-conveying beams", Centre for Offshore Research and Engineering, National University of Singapore, August 2004.

[8] Kuiper G.L. and Metrikine A.V. ' On stability of a clamped-pinned pipe conveying fluid" Heron, Vol.49, No .3, 2004, pp. 211-231.

[9] De Langre E., Paidoussis M.P. , Doaré O. and Modarres-Sadeghi Y., "Flutter of long flexible cylinders in axial flow", Journal of Fluid Mechanics, 2007,V 571, pp. 371-389.

[10] Jing K. and Zhi-Hong Z.,“ Time Prediction Model for Pipeline Leakage Based on Grey Relational Analysis." Physics Procedia, 25, ( 2012 ), pp. $2019-2024$.

[11] Oikonomidis F., Shterenlikht A., and Truma C.E. " Prediction of crack propagation and arrest in X100 natural gas transmission pipelines with a strain rate dependent damage model (SRDD). Part 2: Large scale pipe models with gas depressurization. " International Journal of Pressure Vessels and Piping, (2014), Vol.22, pp. 15-21.

[12] Zhang J., Qiao H.-W., Li P.-Z., Guanghui S. "Prediction of leakage from through pipeline crack" Yuanzineng Kexue Jishu/Atomic Energy 
Science and Technology, April 2015, Vol. 49 (4), pp. 660-666.

[13] Wang X. H., Jiao Y. L., Yang J., Niu Y. C. “The acoustic emission detection and localization technology of the pipeline crack" International Journal of Sensor Networks, January 2016, Vol .20 , No.2, pp.111- 118 .

[14] Kirubakaran A. P., Krishna I. V. M. "Pipeline Crack Detection Using Mathematical Morphological Operator" In book: Knowledge Computing and its Applications, April 2018, Springer, pp. $29-46$.

[15] Mohammed K. I., Raheem H. M, Abdulzhraaalsagheer R. H., Al-Sarraf H., "Detection of faults in pipe that transmits crude oil by vibration sensing: analytically and experimentally" Proceedings of 162nd IASTEM International Conference, Kuwait City, Kuwait, 28, (2018), 29th December.

[16] Razvarz S., Jafari R., Vargas-Jarillo C., Gegov A., Forooshani M. "Leakage Detection in Pipeline Based on Second Order Extended Kalman Filter Observer" IFAC-Papers OnLine, 2019, Volume 52, Issue 29, pp. 116-121.

[17] Lu H., Iseley T., Behbahani S., Fu L. "Leakage detection techniques for oil and gas pipelines: State-of-the-art" Tunnelling and Underground Space Technology, Volume 98, April 2020.

\section{Nomenclature}

A Internal Cross-section area of the pipe, $\left(\mathrm{m}^{2}\right)$

$D \quad$ Outer diameter of the pipe, $(m)$

$D_{i} \quad$ Inner diameter of the pipe, $(m)$

$E \quad$ Modulus of elasticity of the pipe metal, $\left(N / m^{2}\right)$

$F \quad$ Fluid pressure force per unit length applied on the fluid element by the tube, $(\mathrm{N} / \mathrm{m})$

$F^{*}\left(x^{*}\right)$ Dimensionless exciting force parameter, $F^{*}\left(x^{*}\right)=\frac{F(x)}{E I D / L^{4}}$

$f(x . t) \quad$ External force per unit, $(\mathrm{N} / \mathrm{m})$

I Second moment of the area of the pipe cross-section, $\left(\mathrm{m}^{4}\right)$

$K_{1} \quad$ Dimensionless stiffness parameter at crack, $K_{1}=\frac{K_{r c} L}{E I}$
$K_{2} \quad$ Dimensionless stiffness parameter at right hand hinged supported, $K_{2}=\frac{K_{r l} L}{E I}$

$K_{r c} \quad$ Stiffness of rotational spring at crack, (N.m/rad)

$K_{r l} \quad$ Stiffness of rotational spring right hand supported, $(\mathrm{N} . \mathrm{m} / \mathrm{rad})$

$L \quad$ Length of pipe, $(m)$

$M \quad$ The moment at the right hand support of the pipe, (N.m)

$m_{f} \quad$ Fluid mass per unit length, $(\mathrm{kg} / \mathrm{m})$

$m_{p} \quad$ Pipe mass per unit length, $(\mathrm{kg} / \mathrm{m})$

$m_{t} \quad$ Total mass of pipe and fluid per unit length, $(\mathrm{kg} / \mathrm{m})$

$P \quad$ Fluid flow pressure inside the pipe, $\left(\mathrm{N} / \mathrm{m}^{2}\right)$

$Q \quad$ Transverse shear force in the pipe, $\left(N / \mathrm{m}^{2}\right)$

$q$ Shear stress on the internal surface of the pipe, $\left(N / m^{2}\right)$

$r \quad$ Dimensionless mass parameter, $r=\frac{m_{t}}{m_{f}}$

$S \quad$ Inner perimeter of the pipe, $(m)$

$T \quad$ Longitudinal tension in the, $(N)$

$T_{e f f} \quad$ Effective tension in the pipe, $(N)$

$t \quad$ Time, $(s)$

$U \quad$ Velocity of the fluid in $\mathrm{x}$ direction, $(\mathrm{m} / \mathrm{s})$

$w \quad$ Transverse deformation of the pipe, $(m)$

$x \quad$ Axial coordinate, $(m)$

$y \quad$ Transverse coordinate, $(m)$

$S_{t} \quad$ Strouhal number, $S_{t}=\frac{\omega L}{U}$

$V \quad$ Dimensionless velocity parameter, $V=\frac{m_{f} U^{2} L^{2}}{E I}$

$\beta \quad$ Dimensionless axial force parameter, $\beta=\frac{L^{2} T_{e f f}}{E I}$

$\delta x \quad$ Small element in $\mathrm{x}$ direction, $(m)$

$\omega \quad$ External Forced Frequency, $(\mathrm{rad} / \mathrm{s})$

$\rho \quad$ Density of the fluid, $\left(\mathrm{kg} / \mathrm{m}^{3}\right)$

$\rho_{s} \quad$ Density of pipe material, $\left(\mathrm{kg} / \mathrm{m}^{3}\right)$ 